\title{
"Colloidal Life"?
}

\section{Colloquium: Toward living matter with colloidal particles}

Authors: Zorana Zeravcic, Vinothan N. Manoharan, and Michael P. Brenner

Reviews of Modern Physics, v. 89, n. 3, 031001, 2017

\section{Recommended with a Commentary by Alexander Y. Grosberg, Department of Physics and Center for Soft Matter Research, New York University, 726 Broadway, New York, NY 10011}

There has been a remarkable progress in recent years in the attempts to realize selfassembly of increasingly complex structures of colloidal particles. These works are now nicely reviewed by Z.Zeravcic, V.Manoharan, and M.Brenner. The program is, of course, super ambitious: one would like to reproduce with colloids many processes which we intuitively perceive as being characteristic of life, of living systems only, such as self-reproduction, emergence of metabolism, etc. Wisely, authors avoid discussing what is life and what is not life, and instead ask what they can build in the lab that resembles life, or certain aspects of life. Clearly, this approach is also not free of dangers, because if someone wants to reproduce such properties of life as active motion and/or metabolism, then a car might seem like an acceptable model. But what authors present with colloids is nothing short of remarkable.

For "colloids", authors adopt the simplest model - solid spherical particles of micron range size, subject to Brownian motion. First they consider a non-specific short-ranged depletion attraction, easily realized in the lab by adding suitable "depletant" molecules (usually PEG or a similar polymer). Then they switch to more sophisticated specific attraction, which is realized by decorating colloid surfaces with grafted DNA chains with sticky ends. Finally they consider that this short range attraction forces may depend on time in a prescribed way.

They build on the recent advances in exact exhaustive enumeration of possible tight packings of $N$ identical spheres, up to $N=14$ [1]. This set of possible clusters is viewed as the configuration space for the subsequent statistical mechanics consideration. Furthermore, by making a plausible assumption on possible ways to jump from one cluster structure to another, authors transform this set of points into a graph on which they can then build an energy landscape. The richness of the resulting model may seem surprising, but it is extremely impressive nevertheless.

There are many directions to develop these ideas further, but there seems to be also a question pertaining to the foundation of the approach which deserves some attention. I would call it "entropy of mistakes". What I mean is this. Consider first the simplest model with non-specific short range (depletion) attraction between spheres. If attraction energy $\epsilon$ is larger than $k_{B} T$, only clusters with maximal number of contacts can exist. But, as 
the number of spheres in the cluster $N$ increases, it seems increasingly possible to sacrifice parts of the contact energies to gain some fluctuation freedom; in other words, the margin of inequality $\epsilon \gg k_{B} T$ may - or may not - be dependent on $N$. In a similar spirit, for DNA decorated colloids, even with the maximal alphabet, and even if all "right" contacts have the same energy $-\epsilon$, it is not clear that for any realistic model all "wrong" contacts have the same energy. If the number of beads, and, therefore, the number of bead species increases, one would seem to need an increasingly long DNA sticky ends to maintain selectivity. It seems possible (inevitable?) that some of the "wrong" contacts may become less wrong than others or even partly "right" (favorable, with negative energy, perhaps a fraction of $-\epsilon$ ): "all animals are equal, but some animals are more equal than others". Again, corresponding bound may or may not be dependent on $N$; for instance, if we have $Q$ types of beads and $Q^{N}$ possible cluster compositions, while $P^{N}$ clusters can geometrically exist, then $Q>P$ condition (which is a necessary but not sufficient condition to encode one structure by the given set of beads) is $N$-independent. However, if protein folding analogy is of any instructive value, there could be other criteria which scale with cluster surface, not volume, as $R^{N^{2 / 3}}$ [2], and this would lead to an $N$-dependent bound. Also by the same analogy with protein folding, spectral gap might be more informative than the ground state energy itself [3]. Surely, authors are well aware of the entropy problems and pay much attention to the role of competing local minima in both thermodynamics and kinetics, but it might be also worth looking not only at the minima, but at their vicinities. All these questions are interesting (and challenging) for a theorist, but may well be purely academic, given the already demonstrated richness of possibilities at the modest values of $N$.

In any case, I can only recommend reading and re-reading the review by Z.Zeravcic, V.Manoharan, and M.Brenner.

\section{References}

[1] M.C.Holmes-Cerfon, SIAM Rev. 58, 229 (2016).

[2] A.V.Finkelstein, O.V.Galzitskaya, Physics of Life Reviews 1 (1), 23-56 (2004).

[3] E.I.Shakhnovich, Chemical Reviews, 106 (5), 1559 (2006). 